\title{
On Spontaneous Emission Into Guided Modes with Curved Wavefronts
}

\author{
AMNON YARIV AND SHLOMO MARGALIT
}

\begin{abstract}
The problem of spontaneous emission into guided modes with curved wavefronts is examined quantum mechanically. A classical result due to Petermann, which shows an increased emission rate relative to modes with planar phase fronts, is corroborated.
\end{abstract}

$\mathrm{T}$ HE amount of power which is emitted spontaneously into a laser mode plays an important role in determining the spectral and dynamic features of the laser radiation. It figures prominently in semiconductor lasers in questions such as the resonance peak ("spiking resonance") in the modulation response as well as in the number of longitudinal modes which oscillate [2].

An important result due to Petermann [3] is that the spontaneous emission power into modes with curved wavefronts (such as result from gain guiding or a combination of gain and (anti) real-index guiding) is enhanced relative to that emitted into modes in pure index guiding.

At first glance, this result seems to contradict [4] the basic quantum mechanical relationship which states that the ratio of stimulated to spontaneous emission rates into a (any) mode is equal to the number of quanta in the mode. This last statement would suggest that, all other factors remaining the same, the spontaneous emission rates into curved wavefront modes and those with planar wavefronts are equal. This apparent contradiction is not resolved by a study of Petermann's paper [3], which treats the electron in a semiconductor as a localized classical dipole. This classical approach which, in our opinion, still needs to be justified, does not make any contact with the conventional quantum mechanical derivation of the spontaneous emission [5] rate.

To resolve this problem, we undertook a quantum mechanical derivation of the spontaneous emission problem into a mode with a field dependence

$$
E(x, z)=E_{o} \exp \left[-\frac{x^{2}}{2 \omega^{2}}(1+i \chi)-i \beta z\right]
$$

corresponding to a Gaussian beam height $\omega[2]$ and a radius of curvature of the wavefront $R=2 \pi n \omega^{2} / \chi \lambda$. In a real-index guided mode, the astigmatism factor $\chi$ is zero, while in the case of pure gain guiding, $\chi=1$. Higher values of $\chi$ obtain in a combination of anti-index guiding and gain guiding [3]. The key idea is that when $\chi \neq 0$, the field (1) does not qualify as a quantum mechanical mode. A "proper" mode must be an eigenfunction of a field Hamiltonian $\hat{H}_{\text {field }}$ with the total $\hat{H}$ of the form

$$
\hat{H}=\hat{H}_{\text {field }}+\hat{H}_{\text {electron }}+\hat{H}_{\text {interaction }} \text {. }
$$

Manuscript received July 6,1982 . This work was supported by the Office of Naval Research and the Air Force Office of Scientific Research.

The authors are with the California Institute of Technology, Pasadena, CA 91125.

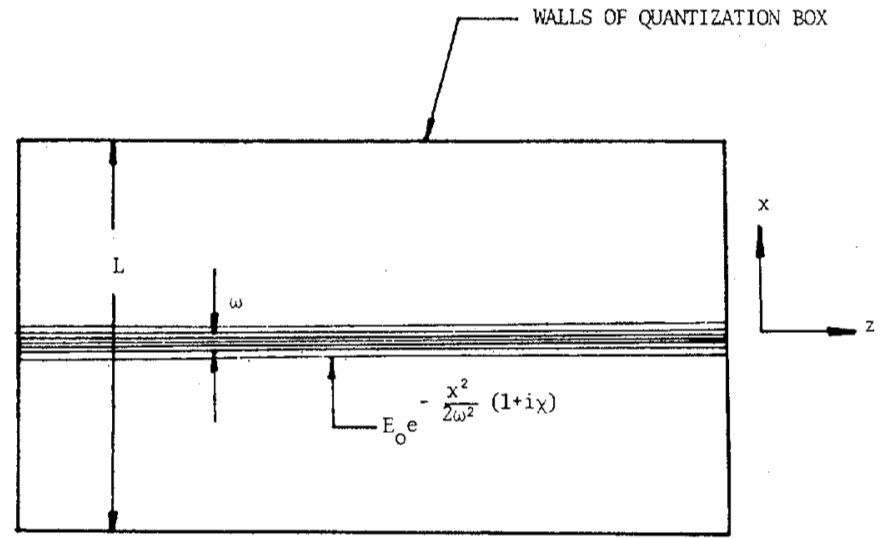

Fig. 1. Schematic representation of the gain guided mode inside an arbitrarily large (height $L$ ) quantization box.

The curved wavefront modes cannot exist without the electrons since they depend for their existence on the presence of of a transverse gain profile [5]. The total mode-electron system is, consequently, not described by a Hamiltonian such as (2). Since $\hat{H}_{\text {field }}$ does not exist independently of $\hat{H}_{\text {electron }}$, we will, consequently, refer to such modes as "improper modes." It follows that the standard quantum mechanical result concerning the ratio of stimulated to spontaneous transition rates does not apply to "improper modes."

To analyze the problem quantum mechanically, we "place" the laser structure inside an arbitrarily large (height $L$ ) quantization box as shown in Fig. 1.

The "big box" modes are of the form $e^{i\left[\omega t-k_{z} z+l(2 \pi / L) x\right]}$ where $k_{z}^{2}+(l(2 \pi / L))^{2}=(2 \pi n / \lambda)^{2}$. The spontaneous emission rate into any one of these modes is given by the standard quantum mechanical result since these modes are defined (and exist) without injected electrons so that the total system Hamiltonian is given by (2).

The calculation of the spontaneous emission rate is thus reduced to that of determining how many "big box" modes are needed to expand the "improper mode" and then summing the spontaneous rates into these modes. The result is the spontaneous transition rate into the "improper mode." Let

$$
\exp \left[-\frac{x^{2}}{2 \omega^{2}}(1+i \chi)\right]=\sum_{-\infty}^{\infty} A_{l} \exp i l \frac{2 \pi}{L} x
$$

from which

$$
A_{l}=\frac{\omega}{L} \sqrt{\frac{2 \pi}{1+i \chi}} \exp -\frac{2 l^{2} \pi^{2} \omega^{2}(1-i \chi)}{L^{2}\left(1+\chi^{2}\right)} .
$$

By approximating the effective number of modes $N \equiv 2 l$ by the value of $l$ where $\left|A_{l}\right|=e^{-1}\left|A_{o}\right|$, we obtain

$$
N=\frac{\sqrt{2}}{\pi} \frac{L}{\omega} \sqrt{1+\chi^{2}} \text {. }
$$


The total spontaneous emission rate into an improper mode is equal to the spontaneous rate into one "big box" mode multiplied by the number $N$ of "big box" modes contained in the improper mode.

Since the spontaneous emission rate into a proper mode is inversely proportional to the mode volume, we have

$$
\left(W_{\text {spont }}\right) \text { into improper mode } \approx A \frac{L}{\omega} \sqrt{1+\chi^{2}} \frac{1}{L}
$$

where $A$ is some constant. The rate of spontaneous emission into a proper real-index guided mode with the same Gaussian beam radius $\omega$ as the improper mode is

$$
\left(W_{\text {spont }}\right) \text { into index guided mode }=A \times\left(\frac{1}{\omega}\right) \text {. }
$$

The factors $1 / L$ and $1 / \omega$ in the last two relations reflect the effective mode heights.

The ratio of the spontaneous emission rates into an "improper mode" and an index guided mode, both with the same beam radius $\omega$, is thus

$$
\frac{\left(W_{\text {spont }}\right) \text { "improper" }}{\left(W_{\text {spont }}\right) \text { index guided }}=\sqrt{1+\chi^{2}} \text {. }
$$

This is the result obtained by Petermann using a classical dipole model for spontaneous transitions.

The rate of stimulated transition into the "improper" and index guided modes with the same $\omega$ is the same when both carry equal power. It follows immediately that the ratio of the rate of induced transition to that of spontaneous transition into an "improper mode" with a constant beam height $\omega$ and astigmatism factor $\chi$ is equal to the number of quanta in the mode divided by $\sqrt{1+\chi^{2}}$. The result is thus fully consistent with quantum mechanics.

\section{REFERENCES}

[1] Y. Suematsu and K. Furuya, "Theoretical spontaneous emission factor of injection lasers," Trans. IECE Japan, vol. E.60, pp. 467$471,1977$.

[2] W. Streifer, D. R. Scifres, and R. D. Burnham, "Longitudinal mode spectra of diode lasers," Appl. Phys. Lett., vol. 40, pp. 305-307, 1982.

[3] K. Petermann, "Calculated spontaneous emission factor for double heterostructure injection lasers with gain-induced wave-guiding," IEEE J. Quantum Electron., vol. QE-15, pp. 566-570, July 1979.

[4] E. Patrak, "Spontaneous emission factor of narrow-stripe gainguided diode lasers," Electron. Lett., vol. 18, pp. 278-279, 1982.

[5] A. Yariv, Quantum Electronics, 2nd ed. New York: Wiley, 1975.

\title{
New Recombination Lasers in $\mathrm{Li}, \mathrm{Al}, \mathrm{Ca}$, and $\mathrm{Cu}$ in a Segmented Plasma Device Employing Foil Electrodes
}

\author{
J. J. MACKLIN, OBERT R. WOOD, II, AND W. T. SILFVAST
}

\begin{abstract}
The use of foil electrodes in an expanding plasma recombination laser device has resulted in the generation of 30 laser transitions in $\mathrm{Li} \mathrm{I}$, Al I, Al II, Al III, Ca I, Ca II, Cu I, and Cu II. The generation of segmented arc plasmas in these metal vapors at the background gas pressures required for laser action had not previously been possible using bulk electrodes, and suggests that conditions necessary for recombination laser action may be obtained for any metal using this technique.
\end{abstract}

A LARGE number of metal-vapor recombination lasers with operating wavelengths from the UV to the mid-IR have recently been demonstrated using a segmented-plasma excitation-recombination (SPER) device [1]-[3]. Both the number and the type of metals studied thus far have been limited, however, by the undesirable occurrence, for some metals, of discharges in the He background gas which shorts out most (or all) of the sequential metal-vapor arcs and reduces (or eliminates) the laser gain. For example, a SPER device employing bulk electrodes (the type of electrodes used in previous SPER devices) of $\mathrm{Li}, \mathrm{Al}, \mathrm{Ca}$, or $\mathrm{Cu}$ cannot be operated at He pressures below about 50 torr. On the other hand, a SPER device employing foil electrodes of these metals can

Manuscript received July 9, 1982.

The authors are with Bell Laboratories, Holmdel, NJ 07733.

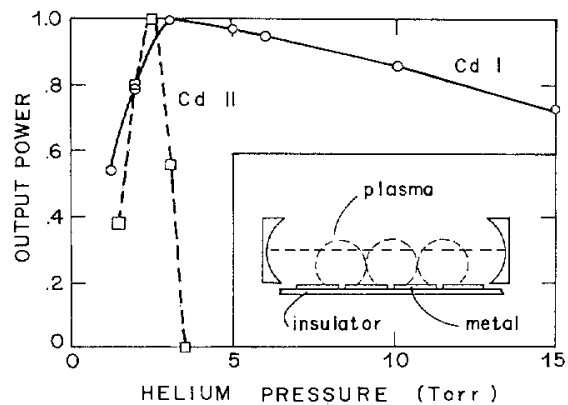

Fig. 1. Relative output power from a Cd SPER laser operating on the $1.433 \mu \mathrm{m}$ transition in Cd I and the $537.8 \mathrm{~nm}$ transition in Cd II as a function of He pressure. Inset shows a highly schematic diagram of the experimental arrangement for a SPER device.

easily be operated at low He pressures where laser action typically occurs (1-10 torr). This new foil electrode device has resulted in 30 new recombination laser transitions in these four metals at wavelengths ranging from 569.6 to $5460 \mathrm{~nm}$. Twenty-eight of these transitions have not previously been observed in laser action. Except for a recent experiment involving the observation of a two-photon laser via optical pumping [4], this letter is the first report of laser action in lithium vapor. Isoelectronic scaling of the new laser transi- 